H. Terao and S. Yuzvinsky

Nagoya Math. J.

Vol. 139 (1995), 129-149

\title{
LOGARITHMIC FORMS ON AFFINE ARRANGEMENTS
}

\author{
HIROAKI TERAO ${ }^{1}$ AND SERGEY YUZVINSKY ${ }^{2}$
}

\section{Introduction}

Let $V$ be an affine of dimension $l$ over some field $\mathbf{K}$. An arrangement $\mathscr{A}$ is a finite collection of affine hyperplanes in $V$. We call $\mathscr{A}$ an $l$-arrangement when we want to emphasize the dimension of $V$. We use [6] as a general reference. Choose an arbitrary point of $V$ and fix it throughout this paper. We will use it as the origin. If $\mathscr{A}$ is central, i.e., $U=\cap_{H \in \mathscr{A}} H \neq \emptyset$, then it is convenient to choose the origin from $U$. Let $S$ be the coordinate ring of $V$ and identity $S$ with the polynomial ring $\mathbf{K}\left[x_{1}, \ldots, x_{l}\right]$, where $x_{1}, \ldots, x_{l}$ are affine coordinates of $V$. For an integer $p, 0 \leq p \leq l$, consider the module of logarithmic differential $p$-forms $\Omega^{p}(\mathscr{A})$ (see 2.1 for definition). We usually simply write $\Omega^{p}=\Omega^{p}(\mathscr{A})$ in this paper. It is a finitely generated $S$-module. Put $\Omega^{p}=0$ if $p<0$ or $p>l$. Now fix a homogeneous polynomial $f \in S$ of a positive degree and define a coboundary operator $d f \wedge$ on $\Omega^{*}=\bigoplus_{p} \Omega^{p}$ by taking the exterior product with $d f$. Then we have a cochain complex, which is also denoted by $\Omega^{*}: \Omega^{*}=\left(\Omega^{*}, d f \wedge\right)$. In this paper, we study its cohomology groups $H^{p}\left(\Omega^{*}\right)$.

Let $L$ be the set of all nonempty intersections of hyperplanes from $\mathscr{A}$. Introduce a partial order to $L$ by reverse inclusion. The poset $L$ is called the intersection poset of $\mathscr{A}$. Let $\mu$ denote the Möbius function on $L$. The characteristic polynomial of $\mathscr{A}$ is

$$
\chi(\mathscr{A}, t)=\sum_{X \in L} \mu(V, X) t^{\mathrm{dim} x}
$$

Our main results are the Theorems 1-5 below.

Theorem 1 (Theorem 7.1). Suppose $f \in S, \operatorname{deg} f=r>0$, satisfies a certain genericity condition with respect to $\mathscr{A}$. Then

Received July 22, 1994

1 Partially supported by National Science Foundation

2 This work was done when the second author was visiting Madison. He is grateful to the Mathematics Department of the University of Wisconsin for its hospitality 
(1) the cohomology groups $H^{p}\left(\Omega^{*}\right)$ are finite dimensional for all $p$,

(2) the Euler characteristic ( = the alternating sum of dimensions of cohomology groups) of the complex $\Omega^{*}$ is equal to $\chi(\mathscr{A}, 1-r)$.

A motivation to study these cohomology groups comes from the study of twisted de Rham complex $\Omega_{\nabla}^{*}=\left(\Omega^{*}, \nabla_{f}\right)$, where the connection map $\nabla_{f}$ is defined by

$$
\nabla_{f}=d+\sum_{j=1}^{n} \lambda_{j} \frac{d \alpha_{j}}{\alpha_{j}} \wedge+d f \wedge
$$

where $d$ is the usual exterior differentiation and $\lambda_{j} \in \mathbf{K}$. In the case of complex field the complex $\Omega_{\nabla}^{*}$ is closely related to the (irregular singular) hypergeometric integrals

$$
\int e^{f(u)} U(u, \lambda, x) d u_{1} \wedge \cdots \wedge d u_{l}
$$

studied in [1] [2]. Theorems 1 and 2 can be regarded as a generalization of results in [4, Proposition 5.10, Theorem 10.3], in which arrangements are assumed to be in general position.

THEOREM 2 (Theorem 7.9). Suppose $f, \operatorname{deg} f=r>0$, satisfies a certain genericity condition with respect to $\mathscr{A}$. Then

(1) the cohomology groups $H^{p}\left(\Omega_{\nabla}^{*}\right)$ are finite dimensional for all $p$,

(2) the Euler characteristic of the complex $\Omega_{\nabla}^{*}$ is equal to $\chi(\mathscr{A}, 1-r)$.

The outline of the paper is as follows. In order to prove Theorem 2 out of Theorem 1, we compare the two complexes $\Omega^{*}$ and $\Omega_{\nabla}^{*}$. In Section 2 , for this purpose, we introduce an increasing filtration $\Omega_{\leq q}^{p}(q \in \mathbf{Z})$ to each $\Omega^{p}$ so that the $E_{1}$-terms of the respective spectral sequences of the filtered complexes $\left\{\Omega_{\leq q}^{*}\right\}$ and $\left\{\left(\Omega_{\nabla}^{*}\right)_{\leq q}\right\}$ coincide.

In Section 3, we define the cone $c \mathscr{A}$ of $\mathscr{A}$. Note that $c \mathscr{A}$ is a central arrangement which is easier to handle than an affine arrangement. We introduce an important short exact sequence (3.4) involving $\Omega^{p}(c \mathscr{A})$.

In Section 4, we introduce the other important short exact sequence (4.1) involving $\mathrm{Gr}_{q} \Omega^{p}(\mathscr{A})=\Omega_{\leq q}^{p} / \Omega_{\leq q-1}^{p}$, which enables us, together with the short exact sequence in Section 3 , to compare $\Omega^{*}(c \mathscr{A})$ and $\Omega^{*}(\mathscr{A})$.

We prove, as a by-product, in Section 5 , the following affine generalization of Solomon-Terao's formula for $\chi(\mathscr{A}, t)[9][6,4.136]$. 
THEOREM 3 (Theorem 5.3).

$$
\lim _{x \rightarrow 1} \sum_{p, q} \operatorname{dim}\left(\operatorname{Gr}_{q} \Omega^{p}(\mathscr{A})\right) x^{q-p}\{t(x-1)-1\}^{p}=\chi(\mathscr{A}, t) .
$$

In Section 6, we compare the cohomology groups $H^{p}\left(\Omega^{*}(c \mathscr{A})\right)$ and $H^{p}\left(\operatorname{Gr} \Omega^{*}(\mathscr{A})\right)$ by using the short exact sequences introduced in Sections 3 and 4. We prove

THEOREM 4 (Theorem 6.9.) Suppose $f \in S, \operatorname{deg} f>0$, satisfies a certain genericity condition with respect to $\mathscr{A}$. Then the following conditions are equivalent for every $p$;

(i) $H^{p+1}\left(\Omega^{*}(c \mathscr{A})\right)=0$,

(ii) $H^{p}\left(\mathrm{Gr} \Omega^{*}(\mathscr{A})\right)=0$.

In Section 7 we prove Theorem 1 using Theorem 3. Then, we "approximate" the cohomology groups $H^{p}\left(\Omega_{\nabla}^{*}\right)$ by $H^{p}\left(\Omega^{*}\right)$ by using a spectral sequence argument and obtain Theorem 2 . Theorems 2 and 4 imply immediately

Theorem 5 (Theorem 7.10). If $H^{p}\left(\Omega^{*}(c \mathscr{A})\right)=0(p \neq l+1)$, then $H^{p}\left(\Omega_{\nabla}^{*}\right)$ $=0(p \neq l)$ and $\operatorname{dim} H^{l}\left(\Omega_{\nabla}^{*}\right)=|\chi(\mathscr{A}, 1-r)|$ for every $f$ of degree $r>0$ which satisfies a certain genericity condition with respect to $\mathscr{A}$.

This theorem can be applied to (1) arrangements in general position (Corollary 7.11), (2) arrangements whose cones are free (Corollary 7.12), and (3) 2 -arrangements (Corollary 7.13).

\section{The module $\Omega^{p}$ of logarithmic forms and its filtration}

Fix an affine arrangement $\mathscr{A}$ in an affine $l$-space $V$ over a field $\mathbf{K}$. Let $S$ be the affine coordinate ring of $V$ and $p$ an integer. We first define the module of logarithmic differential $p$-forms $\Omega^{p}=\Omega^{p}(\mathscr{A})$. For each $H \in \mathscr{A}$ choose $\alpha_{H} \in S$ so that $H=\operatorname{ker}\left(\alpha_{H}\right)$. Let

$$
Q=Q(\mathscr{A})=\prod_{H \in \mathscr{A}} \alpha_{H}
$$

a defining polynomial for $\mathscr{A}$. Let $\Omega^{p}[V]$ denote the $S$-module of all global regular (= polynomial) $p$-forms on $V$ and $\Omega^{p}(V)$ the module of all global rational $p$-forms on $V$. 
Definition 2.1. The module $\Omega^{p}=\Omega^{p}(\mathscr{A})$ of logarithmic $p$-forms with poles along $\mathscr{A}$ is defined as

$$
\Omega^{p}=\Omega^{p}(\mathscr{A})=\left\{\omega \in \Omega^{p}(V) \mid Q \omega \in \Omega^{p}[V] \text { and } Q(d \omega) \in \Omega^{p+1}[V]\right\} .
$$

Put $\Omega^{p}=0$ if $p<0$ or $p>l$.

Remark. The module of logarithmic $p$-forms was first introduced by K. Saito [8] for an arbitrary hypersurface germ in $\mathbf{C}^{l}$. It has been used for the study of central arrangements of hyperplanes [10] [13] [11].

In [6], $\Omega^{p}(\mathscr{A})$ is defined only for central arrangements. However, many results in there remain true for affine arrangements. In fact, in many cases, one can give practically the same proofs. The following two Propositions, which are two of such examples, will be used later.

Proposition 2.2. For each $\omega \in \Omega^{p}(V)$, the following two conditions are equivalent:

(1) $\omega \in \Omega^{p}$,

(2) $Q \omega \in \Omega^{p}[V]$ and $Q\left(d \alpha_{H} / \alpha_{H}\right) \wedge \omega \in \Omega^{p+1}[V]$ for all $H \in \mathscr{A}$.

Proof. Same as Proposition 4.69 in [6].

Proposition 2.3. Let $\Omega^{*}=\bigoplus_{p} \Omega^{p}$. The $S$-module $\Omega^{*}$ is closed under exterior product:

$$
\Omega^{p} \times \Omega^{q} \rightarrow \Omega^{p+q}
$$

Proof. Same as Proposition 4.79 in [6].

It is easy to see that $\Omega^{p}$ is finitely generated $S$-module containing $\Omega^{p}[\mathrm{~V}]$. Also $\Omega^{0}(\mathscr{A})=S$ and $\Omega^{l}(\mathscr{A})=(1 / Q) \Omega^{l}[V]$. When $\mathscr{A}$ is not central, the $S$-modules $\Omega^{p}$ are not naturally graded but we can introduce a natural increasing filtration on them. Let $\beta \in \Omega^{p}[V]$. If each coefficient of $\beta$ is a polynomial of degree at most $q-p$ then we say that the total degree of $\beta$ is $\leq q$ and write $\operatorname{tdeg} \beta$ $\leq q$. Let $\omega \in \Omega^{p}$. It follows from the definition that $\omega$ can be written in the form $\omega=\beta / Q$ where $\beta \in \Omega^{p}[V]$. Let $n=\operatorname{deg} Q=|\mathscr{A}|$. We may formally consider the degree of $1 / Q$ as $-n$ and say that the total degree $\operatorname{tdeg} \omega \leq q$ if $\operatorname{tdeg} \beta \leq q$ $+n$. For example, if $l=1, Q=x(x-1)$, and $\omega=d x / x(x-1)$, then $\operatorname{tdeg} \omega$ $\leq q$ for $q \geq-1$. 
Definition 2.4. Total degree introduces on increasing filtration on $\Omega^{p}$ for $q \in \mathbf{Z}$ by

$$
\Omega_{\leq q}^{p}=\Omega_{\leq q}^{p}(\mathscr{A})=\left\{\omega \in \Omega^{p} \mid \operatorname{tdeg} \omega \leq q\right\} .
$$

Define $\mathbf{K}$-vector spaces for $q \in \mathbf{Z}$ by

$$
\mathrm{Gr}_{q} \Omega^{p}=\operatorname{Gr}_{q} \Omega^{p}(\mathscr{A})=\Omega_{\leq q}^{p} / \Omega_{\leq q-1}^{p} .
$$

If $\mathscr{A}$ is central, then $\Omega^{p}$ is a graded by the total degree, i.e., there exists a decomposition

$$
\Omega^{p}=\bigoplus_{q \in \mathbf{Z}} \Omega_{q}^{p}
$$

with

$$
\Omega_{\leq q}^{p}=\underset{q^{\prime} \leq q}{\bigoplus} \Omega_{q^{\prime}}^{p}
$$

The space $\Omega_{q}^{p}=\Omega_{q}^{p}(\mathscr{A})$ is called the homogeneous part of $\Omega^{p}$ of tdegree $q$.

Definition 2.5. Let $f \in S$. Define maps

$$
d f \wedge: \Omega^{p} \rightarrow \Omega^{p+1}
$$

by sending $\omega \in \Omega^{p}$ to $d f \wedge \omega$. This map is well-defined because of Proposition 2.3. We obtain the cochain complex

$$
\Omega^{*}=\Omega^{*}(\mathscr{A})=\left(\Omega^{*}, d f \wedge\right): \cdots \stackrel{d f \wedge}{\longrightarrow} \Omega^{p} \stackrel{d f \wedge}{\longrightarrow} \Omega^{p+1} \stackrel{d f \wedge}{\longrightarrow} \cdots
$$

Definition 2.6. Suppose $f \in S$ is a polynomial of degree $r(>0)$. Let $q \in \mathbf{Z}$. The cochain complex $\Omega^{*}$ has a subcomplex $\Omega_{\leq q}^{*}=\Omega_{\leq q}^{*}(\mathscr{A})$ which is defined by

$$
\Omega_{\leq q}^{*}=\Omega_{\leq q}^{*}(\mathscr{A}): \cdots \stackrel{d f \wedge}{\longrightarrow} \Omega_{\leq q+(p-l) r}^{p} \stackrel{d f \wedge}{\longrightarrow} \Omega_{\leq q+(p-l+1) r}^{p+1} \stackrel{d f \wedge}{\longrightarrow} \cdots \stackrel{d f \wedge}{\longrightarrow} \Omega_{\leq q}^{l} \rightarrow 0 .
$$

Then this provides an increasing filtration of the cochain complex $\Omega^{*}$. For each $q \in \mathbf{Z}$, define the complex

$$
\mathrm{Gr}_{q} \Omega^{*}=\mathrm{Gr}_{q} \Omega^{*}(\mathscr{A})=\Omega_{\leq q}^{*} / \Omega_{\leq q-1}^{*} .
$$

If $\mathscr{A}$ is central and $f$ is a homogeneous polynomial of degree $r(>0)$, then the cochain complex $\Omega^{*}$ has a subcomplex $\Omega_{q}^{*}=\Omega_{q}^{*}(\mathscr{A})$ which is defined by

$$
\Omega_{q}^{*}=\Omega_{q}^{*}(\mathscr{A}): \cdots \stackrel{d f \wedge}{\longrightarrow} \Omega_{r+(p-l) r}^{p} \stackrel{d f \wedge}{\longrightarrow} \Omega_{r+(p-l+1) r}^{p+1} \stackrel{d f \wedge}{\longrightarrow} \cdots \stackrel{d f \wedge}{\longrightarrow} \Omega_{q}^{l} \rightarrow 0 .
$$


Then

$$
\Omega_{\leq q}^{*}=\underset{q^{\prime} \leq q}{\bigoplus} \Omega_{q^{\prime}}^{*}
$$

Next consider a slightly different cochain complex $\Omega_{\nabla}^{*}$.

Definition 2.7. Let $\lambda_{H} \in \mathbf{K}$ for each $H \in \mathscr{A}$. Define maps $\nabla_{f}: \Omega^{p} \rightarrow \Omega^{p+1}$

$$
\nabla_{f}(\omega)=d \omega+\sum_{H \in \mathscr{A}} \lambda_{H} \frac{d \alpha_{H}}{\alpha_{H}} \wedge \omega+d f \wedge \omega, \quad \omega \in \Omega^{p}
$$

where $d$ is the exterior differentiation. This map is well-defined because $\Omega^{*}$ is closed with respect to exterior product and exterior differential. We obtain the cochain complex

$$
\Omega_{\nabla}^{*}=\Omega_{\nabla}^{*}(\mathscr{A}): \cdots \stackrel{\nabla_{f}}{\longrightarrow} \Omega^{p} \stackrel{\nabla_{f}}{\longrightarrow} \Omega^{p+1} \stackrel{\nabla_{f}}{\longrightarrow} \cdots .
$$

Observe that while $d f \wedge$ is an $S$-linear operator, $\nabla_{f}$ is only $\mathbf{K}$-linear.

Definition 2.8. Let $f \in S$ have degree $r(>0)$ and let $q \in \mathbf{Z}$. Then we can define an increasing filtration of the cochain complex $\Omega_{\nabla}^{*}$ in the same manner as in the case of $\Omega^{*}$ in Definition 2.6. In other words, subcomplexes $\left(\Omega_{\nabla}^{*}\right)_{\leq q}=$ $\left(\Omega_{\nabla}^{*}\right)_{\leq q}(\mathscr{A})$ are defined by

$$
\left(\Omega_{\nabla}^{*}\right)_{\leq q}: \cdots \stackrel{\nabla_{f}}{\longrightarrow} \Omega_{\leq q+(p-l) r}^{p} \stackrel{\nabla_{f}}{\longrightarrow} \Omega_{\leq q+(p-l+1) r}^{p+1} \stackrel{\nabla_{f}}{\longrightarrow} \cdots \stackrel{\nabla_{f}}{\longrightarrow} \Omega_{\leq q}^{l} \rightarrow 0 .
$$

For each $q \in \mathbf{Z}$, define the complex

$$
\mathrm{Gr}_{q} \Omega_{\nabla}^{*}=\operatorname{Gr}_{q} \Omega_{\nabla}^{*}(\mathscr{A})=\left(\Omega_{\nabla}^{*}\right)_{\leq q} /\left(\Omega_{\nabla}^{*}\right)_{\leq q-1} .
$$

The following observation is easy but important:

Lemma 2.9 .

$$
\mathrm{Gr}_{q} \Omega_{\nabla}^{*}=\mathrm{Gr}_{q} \Omega^{*}, \quad q \in \mathbf{Z}
$$

Proof. Note that $d f \wedge$ is the part of the highest degree in the definition of $\nabla_{f}$ because $f>0$. The result is now obvious from definition.

Remark. This shows that the spectral sequences of these two filtered complexes share the same $E_{1}$-term. If the both spectral sequences degenerate at 
$E_{1}$-term, then we have $H^{p}\left(\Omega^{*}\right) \simeq H^{p}\left(\Omega_{\nabla}^{*}\right)$.

\section{Splitting of $\Omega^{*}$ for central arrangements}

Only in this section, let $\mathscr{A}$ denote a central $(l+1)$-arrangement in $V$. Let $x_{0}$, $x_{1}, \ldots, x_{l}$ be linear coordinates for $V$.

First we need to recall the $S$-module $D(\mathscr{A})$ dual to $\Omega^{1}(\mathscr{A})$ (see $[6,4.1]$ ). Let $\operatorname{Der}_{\mathbf{K}}(S)$ be the (free) $S$-module of all derivations of $S$ over $\mathbf{K}$. Then

$$
D(\mathscr{A})=\left\{\theta \in \operatorname{Der}_{\mathbf{K}}(S) \mid \theta(Q) \in Q S\right\} .
$$

While $D(\mathscr{A})$ can be defined in the same way for all arrangements in the case of central arrangement $D(\mathscr{A})$ has a very important element $\theta_{E}$, called the Euler derivation and defined by

$$
\theta_{E}=\sum_{i=1}^{l} x_{\imath} \partial / \partial x_{i}
$$

Let $1 \leq p \leq l$. There exists an $S$-bilinear map (called interior product)

$$
\langle,\rangle: D(\mathscr{A}) \times \Omega^{p}(\mathscr{A}) \rightarrow \Omega^{p-1}(\mathscr{A})
$$

with the following properties (see $[6,4.73,4.74]$ )

(1) $\left\langle\theta, \omega_{1} \wedge \omega_{2}\right\rangle=\left\langle\theta, \omega_{1}\right\rangle \wedge \omega_{2}+(-1)^{p} \omega_{1} \wedge\left\langle\theta, \omega_{2}\right\rangle$,

(2) $\langle\theta, d f\rangle=\theta(f)$

for all $\theta \in D(\mathscr{A}), f \in S, \omega_{1} \in \Omega^{p}(\mathscr{A})$, and $\omega_{2} \in \Omega^{q}(\mathscr{A})$. We will often use the properties (1) and (2) without reference.

Now assume that $\mathscr{A}$ contains the hyperplane $H_{0}=\operatorname{ker}\left(x_{0}\right)$ and let $p$ be an integer. Define two $S$-module homomorphisms

$$
\phi_{p}: \Omega^{p} \rightarrow \Omega^{p-1}, \text { and } \delta_{p}: \Omega^{p} \rightarrow \Omega^{p+1}
$$

via $\phi_{p}(\omega)=(-1)^{p-1}\left\langle\theta_{E}, \omega\right\rangle$ and $\delta_{p}(\omega)=(-1)^{p} \frac{d x_{0}}{x_{0}} \wedge \omega$. We will often omit the indexes from the notation.

The following equalities can be checked easily ((3.2) is sometimes called H. Cartan's homotopy formula):

$$
\begin{gathered}
\phi^{2}=0, \delta^{2}=0, \\
\phi \delta+\delta \phi=\mathrm{id}_{\Omega^{p}} .
\end{gathered}
$$


The equalities (3.1) and (3.2) imply in particular that the following sequence is split exact

$$
0 \rightarrow K^{p} \rightarrow \Omega^{p} \stackrel{\delta}{\rightarrow} K^{p+1} \rightarrow 0
$$

where $K^{p}=\operatorname{ker}\left(\delta_{p}\right)$ and the splitting can be given by the restriction of $\phi_{p+1}$ to $K^{p+1}$.

Clearly both mappings $\phi$ and $\delta$ preserve the (total degree) grading $\Omega^{p}=$ $\bigoplus_{q} \Omega_{q}^{p}$ where $\Omega_{q}^{p}$ is the homogeneous part of tdegree $q$ of $\Omega^{p}(\mathscr{A})$. Thus $K^{p}=$ $\bigoplus_{q} K_{q}^{p}$ is a homogeneous submodule of $\Omega^{p}$ and (3.3) produces for every $q$ the exact sequence of linear spaces

$$
0 \rightarrow K_{q}^{p} \rightarrow \Omega_{q}^{p} \stackrel{\delta}{\rightarrow} K_{q}^{p+1} \rightarrow 0 .
$$

Now fix a homogeneous polynomial $f \in S$ of degree $r(r>0)$ and consider the cochain complex $\Omega^{*}=\left(\Omega^{*}, d f \wedge\right)$ defined in the previous section. Clearly $\delta$ commutes with $d f \wedge$ whence $K^{*}=\left(K^{*}, d f \wedge\right)$ is a subcomplex of $\Omega^{*}=$ $\left(\Omega^{*}, d f \wedge\right)$ and (3.3) generates the exact sequence

$$
\cdots \rightarrow H^{p}\left(K^{*}\right) \rightarrow H^{p}\left(K^{*}\right) \rightarrow H^{p}\left(\Omega^{*}\right) \rightarrow H^{p+1}\left(K^{*}\right) \rightarrow H^{p+1}\left(K^{*}\right) \rightarrow \cdots .
$$

Proposition 3.1. For every $p$ the connecting homomorphism $\gamma: H^{p}\left(K^{*}\right) \rightarrow$ $H^{p}\left(K^{*}\right)$ in (3.5) is the action of $\bar{f}=(-1)^{p-1} r f$ on the $S$-module $H^{p}\left(K^{*}\right)$.

Proof. Let $\omega \in K^{p}, d f \wedge \omega=0$, and $[\omega]$ is the class of $\omega$ in $H^{p}\left(K^{*}\right)$. Since $\phi$ splits the homomorphism $\delta: \Omega^{p-1} \rightarrow K^{p}$ one can view $\gamma([\omega])$ as the class of $d f \wedge \phi(\omega)$. Now we have

$$
\begin{aligned}
d f \wedge \phi(\omega)=(-1)^{p-1} d f & \wedge\left\langle\theta_{E}, \omega\right\rangle=(-1)^{p-1}\left(\left\langle\theta_{E}, d f \wedge \omega\right\rangle+\left\langle\theta_{E}, d f\right\rangle \omega\right) \\
& =(-1)^{p-1} \theta_{E}(f) \omega=\bar{f} \omega
\end{aligned}
$$

which completes the proof.

Finally let $f \in S$ be a homogeneous polynomial of degree $r$. Recall that $d f \wedge$ is homogeneous of degree $+r$ with respect to the grading by total degree and that the complex $\Omega^{*}$ is the direct sum of the complexes $\Omega_{q}^{*}$ where $\Omega_{q}^{*}$ consists of the linear spaces $\Omega_{q+(p-l) r}^{*}$. Similarly $K^{*}=\bigoplus_{q} K_{q}^{*}$ and (3.5) generates for every $q$ the analogous exact sequence of the cohomology spaces of complexes $\Omega_{q}^{*}$ and $K_{q}^{*}$ :

$$
\cdots \rightarrow H^{p}\left(K_{q-1}^{*}\right) \rightarrow H^{p}\left(K_{q}^{*}\right) \rightarrow H^{p}\left(\Omega_{q}^{*}\right) \rightarrow H^{p+1}\left(K_{q}^{*}\right) \rightarrow H^{p+1}\left(K_{q+1}^{*}\right) \rightarrow \cdots
$$




\section{Restriction of forms to affine arrangement}

In the rest of the paper $\mathscr{A}$ is an affine $l$-arrangement in an affine space $V$ over a field $\mathbf{K}$. Let $x_{1}, \ldots, x_{l}$ be affine coordinates. Let $c V$ be an affine $(l+1)$ space with coordinates $x_{0}, x_{1}, \ldots, x_{l}$. Regard $V$ as an affine hyperplane of $c V$ defined by $x_{0}=1$. Let $S^{c}=\mathbf{K}\left[x_{0}, x_{1}, \ldots, x_{l}\right]$ be the coordinate ring of $c V$.

For each $g \in S$ define the homogenization of $g$ as

$$
g^{h}=x_{0}^{\operatorname{deg} g} g\left(x_{1} / x_{0}, \ldots, x_{l} / x_{0}\right)
$$

where deg $g$ is the degree of $g$. Clearly $g^{h} \in S^{c}$ and is homogeneous of the degree $g$. If $s \in S^{c}$ then we put $\varepsilon(s)=s\left(x_{0}=1\right)$. Clearly $\varepsilon: S^{c} \rightarrow S$ is a ring homomorphism and $\varepsilon\left(g^{h}\right)=g$ for every $g \in S$. There is a unique $\varepsilon$-homomorphism of the $S^{c}$-module $\Omega^{p}(c V)$ to the $S$-module $\Omega^{p}(V)$ that maps $d x_{i_{1}} \wedge \cdots \wedge d x_{i_{p}}$ to itself if none of the indexes $i_{j}$ is 0 and to 0 otherwise. We denote this homomorphism also by $\varepsilon$.

Definition 4.1. Let $\mathscr{A}=\left\{H_{1}, \ldots, H_{n}\right\}$ and $Q=\Pi_{i=1}^{n} \alpha_{i}$ be a defining polynomial for $\mathscr{A}$. The cone $c \mathscr{A}$ of $\mathscr{A}$ is defined as the central arrangement in $c V$ given by the polynomial $Q^{c}=x_{0} Q^{h}=x_{0} \Pi_{i=1}^{n} \alpha_{i}^{h}$.

The goal of this section is to relate the modules of logarithmic forms on $c \mathscr{A}$ and $\mathscr{A}$. For that we put $\Omega^{*}=\Omega^{*}(c \mathscr{A})$ and use the notation of the previous section for the differentials and submodules of $\Omega^{*}$.

Definition 4.2. Let $\omega \in K^{p+1}$. Call the restriction of $\omega$ to $\mathscr{A}$ the form

$$
\rho(\omega)=\varepsilon \phi(\omega) \text {. }
$$

Proposition 4.3. (i) $\rho(\omega) \in \Omega^{p}(\mathscr{A})$ for every $\omega \in K^{p+1}$.

(ii) $\rho: K^{p+1} \rightarrow \Omega^{p}(\mathscr{A})$ is an $\varepsilon$-homomorphism of an $S^{c}$-module to an $S$-module.

(iii) The homomorphism $\rho$ is surjective.

(iv) $\operatorname{ker} \rho=\left(x_{0}-1\right) K^{p+1}$.

Proof. (i) Let $\omega \in K^{p+1}$. Clearly $Q \rho(\omega) \in \Omega^{p}[V]$. Now let $i \in\{1, \ldots, n\}$. Since $\phi(\omega) \in \Omega^{p}(\mathscr{A})$ we know, by Proposition 2.2 , that $\alpha_{i}^{h}$ divides $d \alpha_{i}^{h} \wedge$ $Q^{c} \phi(\omega)$. Applying $\varepsilon$ to the coefficients of the latter form we see that $\alpha_{i}$ divides $d \alpha_{i} \wedge Q \rho(\omega)$ which proves the statement again by Proposition 2.2.

(ii) Clear by definition of $\rho$. 
(iii) Let

$$
\eta=\sum_{1 \leq i_{1}<\cdots<i_{p} \leq l} \frac{f_{i_{1}, \ldots, i_{p}}}{Q} d x_{i_{1}} \wedge \cdots \wedge d x_{i_{p}} \in \Omega^{p}(\mathscr{A}) .
$$

Denote by $m$ the maximal degree of the polynomials $f_{I}$ where $I=\left(i_{1}, \ldots, i_{p}\right)$ and define $\tilde{f}_{I}$ as the homogeneous polynomial from $S^{c}$ of degree $m$ such that $\varepsilon\left(\tilde{f}_{I}\right)=f_{I}$. Now put

$$
\omega=\frac{d x_{0}}{x_{0}} \wedge \sum_{I} \frac{\tilde{f}_{I}}{Q^{h}} d x_{I}
$$

where $d x_{I}=d x_{i_{1}} \wedge \cdots \wedge d x_{i_{p}}$.

Our goal is to prove that $\omega \in \Omega^{p+1}(c \mathscr{A})$. Clearly $Q^{c} \omega \in \Omega^{p+1}[c V]$ and $d x_{0} \wedge$ $\omega=0$. Let $i \in\{1, \ldots, n\}$. We know, by Proposition 2.2, that $\alpha_{i}$ divides the form $d \alpha_{i} \wedge Q \eta$, i.e., $\alpha_{i}$ divides every coefficient $g_{J}$ of $d x_{J}=d x_{j_{1}} \wedge \cdots \wedge d x_{j_{p+1}}$ in this form. This implies that $\alpha_{i}^{\mathrm{h}}$ divides $g_{J}^{h}$. Notice that $g_{J}^{h}$ is a linear combination over $\mathbf{K}$ of the coefficients $f_{I}$ of $Q \eta$. Let us compare $g_{J}^{h}$ with the respective coefficient $q_{J}$ of $d x_{0} \wedge d x_{j_{1}} \wedge \cdots \wedge d x_{j_{p+1}}$ in the form $d \alpha_{i}^{h} \wedge Q^{c} \omega$. Notice that both $g_{J}^{h}$ and $q_{J}$ are homogeneous polynomials having the same images under $\varepsilon$ and $\operatorname{deg}\left(q_{J}\right) \geq$ $\operatorname{deg}\left(g_{J}^{h}\right)$. Thus $q_{J}=x_{0}^{s} g_{J}^{h}$ for some non-negative integer $s$. This implies that $\alpha_{i}^{h}$ divides $d \alpha_{i}^{h} \wedge Q^{c} \omega$ and $\omega \in \Omega^{p+1}(c \mathscr{A})$ by Proposition 2.2 .

To complete the proof one should notice that $\delta(\omega)=0$ by construction and check that $\rho(\omega)= \pm \eta$.

(iv) Clearly $\left(x_{0}-1\right) K^{p+1} \subset \operatorname{ker}(\rho)$. Suppose that $\eta \in K^{p+1}$ and $\rho(\eta)=0$. Represent $\phi(\eta)=d x_{0} \wedge \eta_{1}+\eta_{2}$ where $\eta_{1}$ and $\eta_{2}$ are forms without $d x_{0}$ and such that $Q^{c} \eta_{i} \in \Omega^{*}[c V](i=1,2)$. Since $\rho(\eta)=0$ we have by definition of $\rho$ that $\eta_{2}=\left(x_{0}-1\right) \eta_{3}$ where again $Q^{c} \eta_{3} \in \Omega^{*}[c V]$. On the other hand we have $\eta=$ $\delta \phi(\eta)+\phi \delta(\eta)=\delta \phi(\eta)=\delta \eta_{2}=\left(x_{0}-1\right) \delta \eta_{3}$. One checks easily that $\delta \eta_{3} \in$ $K^{p+1}$ which completes the proof.

Remark. 4.4. For any $\eta \in \Omega^{p}(\mathscr{A})$ represent it as in the proof of (iii). Let $q=$ $m-n+p$. Then we have tdeg $\eta \leq q$ and the proof of (iii) shows that there exists $\omega \in K_{q}^{p+1}$ such that $\rho(\omega)=\eta$.

Corollary 4.5. Consider the structure of $S^{c}$-module on $S$ given by $\varepsilon$. Then for every $p$, Proposition 4.3 defines the isomorphism of the following $S$-modules

$$
\Omega^{p}(\mathscr{A}) \simeq K^{p+1} \otimes_{S^{c}} S .
$$


We will also need a graded version of Proposition 4.3. Recall the increasing filtration in $2.4 \cdots \subset \Omega_{\leq q}^{p}(\mathscr{A}) \subset \Omega_{\leq q+1}^{p}(\mathscr{A}) \subset \cdots \subset \Omega^{p}(\mathscr{A})$ where $\Omega_{\leq q}^{p}(\mathscr{A})=$ $\left\{\omega \in \Omega^{p}(\mathscr{A}) \mid \operatorname{tdeg}(\omega) \leq q\right\}$. Also recall the associated graded module $\operatorname{Gr} \Omega^{p}(\mathscr{A})=\bigoplus_{q} \operatorname{Gr}_{q} \Omega^{p}(\mathscr{A})$ where $\operatorname{Gr}_{q} \Omega^{p}(\mathscr{A})=\Omega_{\leq q}^{p}(\mathscr{A}) / \Omega_{\leq q-1}^{p}(\mathscr{A})$.

Now fix $p \in\{0,1, \ldots, l\}$ and $q \in \mathbf{Z}$ and denote by $\pi$ the standard projection $\Omega_{\leq q}^{p}(\mathscr{A}) \rightarrow \mathrm{Gr}_{q} \Omega^{p}(\mathscr{A})$. Clearly the restriction of $\rho$ defines a linear map $K_{q}^{p+1} \rightarrow$ $\Omega_{\leq q}^{p}(\mathscr{A})$ that we still denote by $\rho$. Put $\sigma=\pi \rho: K_{q}^{p+1} \rightarrow \operatorname{Gr}_{q} \Omega^{p}(\mathscr{A})$.

PROPOSITION 4.6. The following sequence is exact

$$
0 \rightarrow K_{q-1}^{p+1} \stackrel{x_{0}}{\rightarrow} K_{q}^{p+1} \stackrel{\sigma}{\rightarrow} \mathrm{Gr}_{q} \Omega^{p}(\mathscr{A}) \rightarrow 0
$$

Proof. The surjectively of $\sigma$ follows from Remark 4.4. It suffices to prove that $\operatorname{ker} \sigma=x_{0} K_{q-1}^{p+1}$. Clearly $x_{0} K_{q-1}^{p+1} \subset \operatorname{ker} \sigma$. Let $\eta \in \operatorname{ker} \sigma$, i.e., $\rho(\eta) \in$ $\Omega_{\leq q-1}^{p}(\mathscr{A})$. Then by the surjectivity of $\sigma$ and Proposition 4.3 (iv) there exist $q^{\prime}<q, \eta^{\prime} \in K_{q^{\prime}}^{p+1}$, and $\eta^{\prime \prime} \in K^{p+1}$ such that $\eta-\eta^{\prime}=\left(x_{0}-1\right) \eta^{\prime \prime}$. If $\eta_{1}$ is the homogeneous component of $\eta^{\prime \prime}$ of maximal degree then $\eta=x_{0} \eta_{1}$ which completes the proof.

\section{A formula for the characteristic polynomials of affine arrangements}

Use the same notation as in the previous section. Let $p$ and $q$ be integers. By (3.4), we have

$$
\operatorname{dim} \Omega_{q}^{p}(\mathscr{A})=\operatorname{dim} K_{q}^{p+1}+\operatorname{dim} K_{q}^{p}
$$

Also, by (4.1), we have

$$
\operatorname{dim} \operatorname{Gr}_{q} \Omega^{p}(\mathscr{A})=\operatorname{dim} K_{q}^{p+1}-\operatorname{dim} K_{q-1}^{p+1} .
$$

Since $\Omega^{*}(\mathscr{A})$ is doubly graded, we can naturally define the Poincaré series $\operatorname{Poin}\left(\Omega^{*}(c \mathscr{A}) ; x, y\right)$ in two variables by

$$
\operatorname{Poin}\left(\Omega^{*}(c \mathscr{A}) ; x, y\right)=\sum_{p, q}\left(\operatorname{dim} \Omega_{q}^{p}(c \mathscr{A})\right) x^{q} y^{p} .
$$

Similarly we can define $\operatorname{Poin}\left(K^{*} ; x, y\right)$ and $\operatorname{Poin}\left(\operatorname{Gr} \Omega^{*}(\mathscr{A}) ; x, y\right)$;

$$
\begin{aligned}
\operatorname{Poin}\left(K^{*} ; x, y\right) & =\sum_{p, q}\left(\operatorname{dim} K_{q}^{p}\right) x^{q} y^{p}, \\
\operatorname{Poin}\left(\operatorname{Gr} \Omega^{*}(\mathscr{A}) ; x, y\right) & =\sum_{p, q}\left(\operatorname{dim} \operatorname{Gr}_{q} \Omega^{p}(\mathscr{A})\right) x^{q} y^{p} .
\end{aligned}
$$


PROPOSITION 5.1.

$$
\operatorname{Poin}\left(\operatorname{Gr} \Omega^{*}(\mathscr{A}) ; x, y\right)=(1-x)(1+y)^{-1} \operatorname{Poin}\left(\Omega^{*}(c \mathscr{A}) ; x, y\right) .
$$

Proof. By (5.1) we have

$$
\operatorname{Poin}\left(\Omega^{*}(c \mathscr{A}) ; x, y\right)=\left(1+y^{-1}\right) \operatorname{Poin}\left(K^{*} ; x, y\right) \text {. }
$$

By (5.2) we have

$$
\operatorname{Poin}\left(\mathrm{Gr} \Omega^{*}(\mathscr{A}) ; x, y\right)=\left(y^{-1}-x y^{-1}\right) \operatorname{Poin}\left(K^{*} ; x, y\right) \text {. }
$$

The following result is essentially due to Solomon-Terao [9] [6, Proposition 4.133, Theorem 4.136]:

TheOREM 5.2. (1) $\Psi(\mathscr{A} ; x, t)=\operatorname{Poin}\left(\Omega^{*}(c \mathscr{A}) ; x, \frac{t(1-x)-1}{x}\right)$ has no pole at $x=1$,

(2) $\Psi(c \mathscr{A} ; 1, t)=\chi(c \mathscr{A}, t)$.

Remark. In [6], the results are stated in terms of pdegree instead of tdegree. This is why they appear slightly different.

The following result is the affine generalization of Theorem 5.2. It contains Theorem 3 in the Introduction.

Theorem 5.3. (1) $\Psi(\mathscr{A} ; x, t):=\operatorname{Poin}\left(\operatorname{Gr} \Omega^{*}(\mathscr{A}) ; x, \frac{t(1-x)-1}{x}\right)$ has no pole at $x=1$,

(2) $\Psi(\mathscr{A} ; 1, t)=\chi(\mathscr{A}, t)$.

Proof. Set $y=x^{-1}(t(1-x)-1)$ in Proposition 5.1. Then we have

$$
\begin{aligned}
\Psi(\mathscr{A} ; x, t) & =\operatorname{Poin}\left(\operatorname{Gr} \Omega^{*}(\mathscr{A}) ; x, x^{-1}(t(1-x)-1)\right) \\
& =\frac{x}{t-1} \operatorname{Poin}\left(\Omega^{*}(c \mathscr{A}) ; x, x^{-1}(t(1-x)-1)\right) \\
& =x(t-1)^{-1} \Psi(c \mathscr{A} ; x, t) .
\end{aligned}
$$

Since $\chi(c \mathscr{A}, t)=(t-1) \chi(\mathscr{A}, t)$ (e.g., [6, Proposition 2.51, Definition 2.52]) Theorem 5.2 completes the proof. 


\section{Relations between cohomology of affine arrangement and its cone}

Use the same notation as the previous section. Fix a polynomial $f \in S$ of degree $r>0$. Let $f^{h} \in S^{c}$ be the homogenization of $f$. As in (3.3), the exterior multiplication by $d f^{h}$ defines a complexes $\left(K^{*}, d f^{h} \wedge\right)=K^{*}$ for the cone $c \mathscr{A}$. Also the exterior multiplication by $d f$ defines another complex $\left(\Omega^{*}(\mathscr{A}), d f \wedge\right)=$ $\Omega^{*}(\mathscr{A})$ as in 2.5. The goal of this section is to relate the cohomology of these complexes.

PROPOSITION 6.1. The homomorphisms $\rho$ define a homomorphism of the complexes $K^{*} \rightarrow \Omega^{*}(\mathscr{A})$ that decreases dimension by 1 .

Proof. It suffices to prove that $\rho$ commutes with the differentials. Let $\omega \in$ $K^{p+1}$. Notice that $\varepsilon(\omega)=0$. Using this we have

$$
\begin{gathered}
\rho\left(d f^{c} \wedge \omega\right)=\varepsilon \phi\left(d f^{c} \wedge \omega\right)=\varepsilon\left((-1)^{p+1} \theta_{E}\left(f^{c}\right) \omega+d f^{c} \wedge \phi(\omega)\right) \\
=\varepsilon\left(d f^{c}\right) \wedge \rho(\omega)=d f \wedge \rho(\omega)
\end{gathered}
$$

that proves the statement.

COROLLARY 6.2. We have the short exact sequence of complexes

$$
0 \rightarrow K^{*} \stackrel{x_{0}-1}{\rightarrow} K^{*} \stackrel{\rho}{\rightarrow} \Omega^{*}(\mathscr{A}) \rightarrow 0
$$

COROLLARY 6.3. We have the following exact sequence of modules

$$
\begin{aligned}
\cdots & \rightarrow H^{p+1}\left(K^{*}\right) \stackrel{x_{0}-1}{\rightarrow} H^{p+1}\left(K^{*}\right) \rightarrow H^{p}\left(\Omega^{*}(\mathscr{A})\right) \\
& \rightarrow H^{p+2}\left(K^{*}\right) \stackrel{x_{0}-1}{\rightarrow} H^{p+2}\left(K^{*}\right) \rightarrow \cdots .
\end{aligned}
$$

COROLlary 6.4. Consider the complex $\Omega^{*}(c \mathscr{A})=\left(\Omega^{*}(c \mathscr{A}), d f^{h} \wedge\right)$ and suppose that $H^{p+1}\left(\Omega^{*}(c \mathscr{A})\right)=0$ for some $p$. Then $H^{p}\left(\Omega^{*}(\mathscr{A})\right)=0$.

Proof. Put $H^{i}=H^{i}\left(K^{*}\right)$ for every $i$. It follows from (3.5) and Proposition 3.1 that the action of $f^{h}$ on $H^{p+1}$ is surjective. Since $H^{p+1}$ is a graded module and $f^{h}$ is homogeneous of positive degree this implies that $H^{p+1}=0$. Since $H^{p+2}$ is also a graded module and $x_{0}$ has a positive degree the action of $x_{0}-1$ on $H^{p+2}$ is injective. Now the result follows from the exact sequence (6.2). 
The rest of the section concerns cohomology of a complex containing more subtle information about $\Omega^{*}(\mathscr{A})$.

Fix an integer $q$ and recall the complex $\operatorname{Gr}_{q}^{*}(\mathscr{A})=\mathrm{Gr}_{q}^{*}$ from Definition 2.6. Denote by $\mathrm{Gr}^{*}$ the direct sum of the complexes $\mathrm{Gr}_{q}^{*}$ for all $q$. Then it follows from Proposition 6.1 that the differentials commute wih $\sigma$ whence (4.1) defines the short exact sequence of complexes

$$
0 \rightarrow K^{*} \stackrel{x_{0}}{\rightarrow} K^{*} \stackrel{\sigma}{\rightarrow} \mathrm{Gr}^{*} \rightarrow 0
$$

The sequence (6.4) induces the exact sequence of cohomology

$$
\cdots H^{p+1}\left(K^{*}\right) \stackrel{x_{0}}{\rightarrow} H^{p+1}\left(K^{*}\right) \rightarrow H^{p}\left(\mathrm{Gr}^{*}\right) \rightarrow H^{p+2}\left(K^{*}\right) \stackrel{x_{0}}{\rightarrow} H^{p+2}\left(K^{*}\right) \rightarrow \cdots
$$

Now we need certain information about the annihilator $\operatorname{Ann}\left(H^{p}\right)$ of the $S^{c}$-module $H^{p}=H^{p}\left(K^{*}\right)$. Define

$$
D_{0}(c \mathscr{A})=\left\{\theta \in D(c \mathscr{A}) \mid \theta\left(x_{0}\right)=0\right\} .
$$

Proposition 6.5. Let $\theta \in D_{0}(\mathscr{A})$. Then $\theta\left(f^{h}\right) \in \operatorname{Ann}\left(H^{p}\right)$ for every $p$.

Proof. Let $\omega \in K^{p}$ and $d f^{h} \wedge \omega=0$. Then we have

$$
0=\left\langle\theta, d f^{h} \wedge \omega\right\rangle=\theta\left(f^{h}\right) \omega-d f^{h} \wedge\langle\theta, \omega\rangle .
$$

Since $\theta \in D(\mathscr{A})$ then $\eta=\langle\theta, \omega\rangle \in \Omega^{p-1}(\mathscr{A})$. To prove the result it suffices to check that $\eta \in K^{p-1}$, i.e., $d x_{0} \wedge \eta=0$. We have

$$
d x_{0} \wedge\langle\theta, \omega\rangle=\theta\left(x_{0}\right) \omega-\left\langle\theta, d x_{0} \wedge \omega\right\rangle
$$

that is 0 since $\theta\left(x_{0}\right)=0$ and $\omega \in K^{p}$.

It suffices for our purposes to use only a particular case of Proposition 6.5. For any $X \in L(c \mathscr{A}) \backslash\{0\}$ put $\pi_{X}=\Pi_{H \notin \mathscr{A}_{X}} \alpha_{H}$. Besides for any nonzero vector $a \in c V$ denote by $\partial_{a}$ the derivation of $S^{c}$ that is the differentiation in the direction of $a$.

COROllary 6.6. For any nonzero $X \in L(\mathcal{A})$ and nonzero vector $a \in X \cap H_{0}$ we have $\pi_{X} \partial_{a} f^{h} \in$ Ann $H^{p}$.

Proof. Due to Proposition 6.5 it suffices to check that $\pi_{X} \partial_{a} \in D_{0}(c \mathscr{A})$. The check is straightforward. 
Definition 6.7. A polynomial $f$ is $\mathscr{A}$-transverse if for every $X \in L(c \mathscr{A})$ the restriction of its homogenization $f^{h}$ to $X$ has no critical points outside the origin.

Note that this is a generic condition for $f$ with a fixed positive degree.

Lemma 6.8. Suppose that $f$ is $\mathscr{A}$-transverse. Let $D_{0}(c \mathscr{A}) f^{h}=\left\{\theta\left(f^{h}\right) \mid \theta \in\right.$ $\left.D_{0}(c \mathscr{A})\right\}$ and $S_{+}^{c}=\left\{g \in S^{h} \mid g(0, \ldots, 0)=0\right\}$.

(1) Let $I_{f}$ be the ideal of $S^{c}$ generated by $f^{c}$ and $D_{0}(c \mathscr{A}) f^{h}$. Then the radical of $I_{f}$ is equal to $S_{+}^{c}$.

(2) Let $I_{x}$ be the ideal of $S^{c}$ generated by $x_{0}$ and $D_{0}(c \mathscr{A}) f^{h}$. Then the radical of $I_{x}$ is equal to $S_{+}^{c}$.

Proof. (1): We can assume that the field $\mathbf{K}$ is algebraically closed by passing to its algebraic closure if necessary. Let $I=I_{f}$ and $V(I)$ the set of zeros of $I$. By the Nullstellensatz it is enough to show that $V(I)$ is contained in $\{0\}$. Take arbitrary $v \in V(I)$ and find the maximal $X \in L(c \mathscr{A})$ such that $v \in X$. Suppose $X \neq$ 0 . Then due to Corollary $6.6, \pi_{X} \partial_{a} f^{h} \in I$ for every vector $a \in X \cap H_{0}$. Since $\pi_{X}(v) \neq 0$ we have $\partial_{a} f^{h}(v)=0$. Since $f$ is $\mathscr{A}$-transverse, the restriction of $f^{h}$ to $X$ cannot have a critical point at $v$. This is a contradiction.

(2) The proof is similar.

Now we are ready to prove one of the main results of the paper. The following theorem is Theorem 4 in the Introduction.

THEOREM 6.9. Let $f$ be $\mathscr{A}$-transverse. Then the following conditions are equivalent for every $p$;

(i) $H^{p+1}\left(\Omega^{*}(c \mathscr{A})\right)=0$,

(ii) $H^{p}\left(\mathrm{Gr}^{*}\right)=0$.

Proof. Consider the exact sequences (3.5) (taking into consideration Proposition 3.1) and (6.5). Since $H^{p+1}\left(K^{*}\right)$ is a graded $S^{c}$-module and both $\bar{f}^{h}$ and $x_{0}$ are homogeneous of positive degrees, each of the conditions (i) and (ii) implies that $H^{p+1}\left(K^{*}\right)=0$. Put $Z=\bigcup_{I \in \operatorname{Ass}(H)} I$ where $H=H^{p+2}\left(K^{*}\right)$. It suffices to prove that $\bar{f}^{h} \in Z$ if and only if $x_{0} \in Z$.

Suppose $I \in \operatorname{Ass}(H)$ and $\bar{f}^{h} \in I$. By Proposition 6.5, $D_{0}(c \mathscr{A}) f^{h} \subseteq I$. Since $f^{h} \in I, I$ is a prime ideal containing $I_{f}$ in Lemma 6.8 (i). Thus $I$ contains $S_{+}^{c}$ and, in particular, $x_{0}$.

Now suppose $I \in \operatorname{Ass}(H)$ and $x_{0} \in I$. Using reasoning similar to the above 
and applying Lemma 6.8 (ii) we conclude that $I$ contains $S_{+}^{c}$ and, in particular, $f^{h}$.

\section{Results on $H^{p}\left(\Omega^{*}\right)$ and $H^{p}\left(\Omega_{\nabla}^{*}\right)$}

Use the same notation as the previous section.

The following theorem contains Theorem 1 in the Introduction. Recall the definition (1.1) of the characteristic polynomial $\chi(\mathscr{A}, t)$.

Theorem 7.1. Suppose $f \in S, \operatorname{deg} f=r>0$, is $\mathscr{A}$-transverse. Then

(1) the cohomology groups $H^{p}\left(\Omega^{*}\right)$ and $H^{p}\left(\mathrm{Gr}^{*}\right)$ are finite dimensional for all $p$,

(2) the Euler characteristics ( = the alternating sum of dimensions of cohomology groups) of the complexes $\Omega^{*}$ and $\mathrm{Gr}^{*}$ are both equal to $\chi(\mathscr{A}, 1-r)$.

Proof. (1): We only need to show that $H^{p}\left(\mathrm{Gr}^{*}\right)$ is finite dimensional. Thanks to the exact sequence (6.5) it is enough to prove that the kernel and the cokernel of the multiplication map of $x_{0}: H^{p}\left(K^{*}\right) \rightarrow H^{p}\left(K^{*}\right)$ are both finite dimensional. Recall $H^{p}\left(K^{*}\right)$ is annihilated by $D_{0}(\mathscr{A}) f^{h}$ by Proposition 6.5 . Thus both the kernel and the cokernel are annihilated by $x_{0}$ and $D_{0}(c \mathscr{A}) f^{h}$. By Lemma 6.8 , the radical of the ideal generated by $x_{0}$ and $D_{0}(c \mathscr{A}) f^{h}$ contains $S_{+}^{c}$. This shows (1).

(2): Let $E_{r}^{p, q}$ be the spectral sequence associated with the filtered complex $\left\{\Omega_{\leq q}^{*}\right\}$. Then $E_{1}^{p, q}=H^{p+q}\left(\mathrm{Gr}_{-p}^{*}\right)=0$ except for finitely many pairs $(p, q)$ by $(1)$. So we have

$$
\begin{aligned}
\sum_{p}(-1)^{p} \operatorname{dim} H^{p}\left(\mathrm{Gr}^{*}\right) & =\sum_{p, q}(-1)^{p+q} \operatorname{dim} E_{1}^{p, q}=\sum_{p, q}(-1)^{p+q} \operatorname{dim} E_{2}^{p, q} \\
& =\cdots \\
& =\sum_{p, q}(-1)^{p+q} \operatorname{dim} E_{\infty}^{p, q}=\sum_{p}(-1)^{p} \operatorname{dim} H^{p}\left(\Omega^{*}\right) .
\end{aligned}
$$

Therefore it is enough to prove the statement for $\mathrm{Gr}^{*}$. Recall Poin $\left(\mathrm{Gr} \Omega^{*}(\mathscr{A}): x, y\right)$ from (5.3) and set $y=-x^{-r}$. Then we get

$$
\begin{aligned}
\operatorname{Poin}\left(\operatorname{Gr} \Omega^{*}(\mathscr{A}): x,-x^{-r}\right) & =\sum_{p, q}\left(\operatorname{dim} \operatorname{Gr}_{q} \Omega^{p}\right) x^{q}\left(-x^{-r}\right)^{p} \\
& =\sum_{m} x^{m-r l} \sum_{p}(-1)^{p}\left(\operatorname{dim} \operatorname{Gr}_{m+(p-l) r} \Omega^{p}\right) \\
& =\sum_{m} x^{m-r l} \sum_{p}(-1)^{p} \operatorname{dim} H^{p}\left(\mathrm{Gr}_{m}^{*}\right)
\end{aligned}
$$

On the other hand, recall 


$$
\Psi(\mathscr{A} ; x, t)=\operatorname{Poin}\left(\operatorname{Gr} \Omega^{*}(\mathscr{A}): x, \frac{t(1-x)-1}{x}\right)
$$

from Theorem 5.3. Set $t=\left(1-x^{1-r}\right)(1-x)^{-1}$ in $\Psi(\mathscr{A} ; x, t)$ and take the limit of $x \rightarrow 1$. Then, applying Theorem 5.3 , we have

$$
\begin{aligned}
\chi(\mathscr{A}, 1-r)=\lim _{x \rightarrow 1} \Psi\left(\mathscr{A} ; x,\left(1-x^{1-r}\right)(1-x)^{-1}\right) & =\lim _{x \rightarrow 1} \operatorname{Poin}\left(\mathrm{Gr} \Omega^{*}(\mathscr{A}): x,-x^{-r}\right) \\
& =\sum_{p} \operatorname{dim} H^{p}\left(\mathrm{Gr}^{*}\right)
\end{aligned}
$$

The following condition (for central arrangements) was introduced, without name, in [7, Definition 5.4]:

Definition 7.2. Let pd denote the projective dimension over $S$. If

$$
\operatorname{pd} \Omega^{p}(\mathscr{A}) \leq p \text { for } 0 \leq p \leq l,
$$

then we say that $\mathscr{A}$ is tame.

The following result is well-known (e.g., see [7, Theorem 5.8]):

Theorem 7.3. Let $S=\mathbf{C}\left[x_{1}, x_{2}, \ldots, x_{l}\right]$ be a polynomial ring. Let

$$
\mathscr{C}^{\cdot}: 0 \rightarrow \mathscr{C}^{0} \stackrel{d_{0}}{\rightarrow} \mathscr{C}^{1} \stackrel{d_{1}}{\rightarrow} \ldots
$$

be a cochain complex of finite $S$-modules. Suppose that each coboundary map $d$ is $S$-linear. Assume that each cohomology group $H^{q}\left(\mathscr{C}^{\circ}\right)$ is finite dimensional over $\mathbf{C}$. If a nonnegative integer $q$ satisfies

$$
\operatorname{pd} \mathscr{C}^{p}<l+p-q \text { for all } p
$$

then $H^{q}\left(\mathscr{C}^{*}\right)=0$.

Theorem 7.4. If $\mathscr{A}$ is tame, then we have $H^{p}\left(\Omega^{*}\right)=0(p \neq l)$ and $\operatorname{dim} H^{l}\left(\Omega^{*}\right)$ $=|\chi(\mathscr{A}, 1-r)|$ for every $\mathscr{A}$-transverse polynomial $f$ of degree $r>0$.

Proof. Since the cohomology groups $H^{p}\left(\Omega^{*}(\mathscr{A})\right)$ are finite dimensional and the differential $d f \wedge$ is $S$-linear, we can apply Theorem 7.3 to conclude $H^{p}\left(\Omega^{*}\right)=0(p \neq l)$. Apply Theorem 7.1.

Definition 7.5. The affine arrangement is called free if the $S$-module $\Omega^{1}(\mathscr{A})$ is a free $S$-module. 
Remark. In [6], the definition of free arrangement is given only for central arrangements. We can show that an affine arrangement $\mathscr{A}$ is free if and only if its localization $\mathscr{A}_{X}:=\{H \in \mathscr{A} \mid X \subseteq H\}$ is a (central) free arrangement for all $X \in$ $L(\mathscr{A})$. We can also show that $\Omega^{p}(\mathscr{A})=\wedge_{S}^{p} \Omega^{1}(\mathscr{A})$ for all $p$ if $\mathscr{A}$ is free.

The following is corollary from Theorem 7.4:

COROLlary 7.6. If an affine l-arrangement $\mathscr{A}$ is free, then we have $H^{p}\left(\Omega^{*}\right)=0$ $(p \neq l)$ and $H^{l}\left(\Omega^{*}\right)=|\chi(\mathscr{A}, 1-\gamma)|$ for every $\mathscr{A}$-transverse polynomial $f$ of degree $r>0$.

We also have the vanishing of the three lowest cohomology groups for any affine arrangements:

Proposition 7.7. Let $f$ be an arbitrary $\mathscr{A}$-transverse polynomial of a positive degree. Then

(1) $H^{0}\left(\Omega^{*}\right)=0$ if $l>0$,

(2) $H^{1}\left(\Omega^{*}\right)=0$ if $l>1$,

(3) $H^{2}\left(\Omega^{*}\right)=0$ if $l>2$.

Proof. Same as the proof of Proposition 5.15 in [7].

Corollary 7.8. If $\mathscr{A}$ is an affine l-arrangement with $l \leq 3$, then we have $H^{p}\left(\Omega^{*}\right)=0(p \neq l)$ and $\operatorname{dim} H^{l}\left(\Omega^{*}\right)=|\chi(\mathscr{A}, 1-r)|$ for every $\mathscr{A}$-transverse polynomial $f$ of degree $r>0$.

Next we finally get results concerning the dimensions of cohomology groups of the complex $\Omega_{\nabla}^{*}$. The following theorem contains Theorem 2 in the Introduction.

Theorem 7.9. Suppose $f \in S, \operatorname{deg} f=r>0$, is $\mathscr{A}$-transverse. Then

(1) the cohomology groups $H^{p}\left(\Omega_{\nabla}^{*}\right)$ and $H^{p}\left(\mathrm{Gr} \Omega_{\nabla}^{*}\right)$ are finite dimensional for all $p$,

(2) the Euler characteristics (= the alternating sum of dimensions of cohomology groups) of the complexes $\Omega_{\nabla}^{*}$ and $\operatorname{Gr} \Omega_{\nabla}^{*}$ are both equal to $\chi(\mathscr{A}, 1-r)$.

Proof. Recall $H^{p}\left(\mathrm{Gr}_{q} \Omega^{*}(\mathscr{A})\right)=H^{p}\left(\mathrm{Gr}_{q} \Omega_{\nabla}^{*}(\mathscr{A})\right)$ from the Remark to 2.9 . Thus we can prove this by the same method as in the proof of Theorem 7.1. 
The following theorem is Theorem 5 in the Introduction.

THEOREM 7.10. Let $f$ be an $\mathscr{A}$-transverse polynomial of degree $r>0$. If the cone $c \mathscr{A}$ of $\mathscr{A}$ satisfies $H^{p}\left(\Omega^{*}(c \mathscr{A})\right)=0$ for all $p \neq l+1$, then $H^{p}\left(\Omega_{\nabla}^{*}\right)=0$ for all $p \neq l$ and $\operatorname{dim} H^{l}\left(\Omega_{\nabla}^{*}\right)=|\chi(\mathscr{A}, 1-r)|$ for every $\mathscr{A}$-transverse polynomial $f$ of degree $r>0$.

Proof. Since $H^{p}\left(\Omega^{*}(c \mathscr{A})\right)=0$ for $p \neq l+1$, we have $H^{p}\left(\mathrm{Gr}_{q} \Omega^{*}(\mathscr{A})\right)=0$ for $p \neq l, q \in \mathbf{Z}$ by Theorem 6.9. Since $H^{p}\left(\operatorname{Gr}_{q} \Omega^{*}(\mathscr{A})\right)=H^{p}\left(\operatorname{Gr}_{q} \Omega_{\nabla}^{*}(\mathscr{A})\right)$ from the Remark to 2.9 , the spectral sequences of filtered complexes $\left\{\Omega_{\leq q}^{*}\right.$ \} and $\left\{\left(\Omega_{\nabla}^{*}\right)_{\leq q}\right\}$ are both $E_{1}$-degenerate. Therefore $\operatorname{dim} H^{p}\left(\Omega^{*}\right)=\operatorname{dim} H^{p}\left(\Omega_{\nabla}^{*}\right)$.

The following result is one of the main results in [4]:

Corollary 7.11. If $\mathscr{A}$ is in general position (for the definition, e.g., see [6, Definition 5.19]), then $H^{p}\left(\Omega_{\nabla}^{*}\right)=0(p \neq l)$ and

$$
\operatorname{dim} H^{l}\left(\Omega_{\nabla}^{*}\right)=|\chi(\mathscr{A}, 1-r)|=\sum_{i=0}^{l}\left(\begin{array}{c}
n \\
l-i
\end{array}\right) q^{i}
$$

for every $\mathscr{A}$-transverse polynomial $f$ of degree $r>0$.

Proof. When $\mathscr{A}$ is in general position, its cone $\mathscr{C A}$ is a generic arrangement (for the definition, e.g., see [6, Definition 5.22]). We have $H^{p}\left(\Omega^{*}(c \mathscr{A})\right)=0(p \neq l$ $+1)$ by Proposition 5.12 in [7]. The characteristic polynomial $\chi(\mathscr{A}, t)$ of $\mathscr{A}$ is directly computed.

COROLlary 7.12. If $\mathscr{A}$ is an affine l-arrangement such that its cone $c \mathscr{A}$ is free with its exponents (for the definition, e.g., see [6, Definition 4.25]) $\left\{1, b_{1}, b_{2}, \ldots\right.$, $\left.b_{l}\right\}$ then $H^{p}\left(\Omega_{\nabla}^{*}\right)=0(p \neq l)$ and

$$
\operatorname{dim} H^{l}\left(\Omega_{\nabla}^{*}\right)=|\chi(\mathscr{A}, 1-r)|=\prod_{i=1}^{l}\left(b_{i}+q\right)
$$

for every $\mathscr{A}$-transverse polynomial $f$ of degree $r>0$.

Proof. Since $c \mathscr{A}$ is free, we have $H^{p}\left(\Omega^{*}(c \mathscr{A})\right)=0(p \neq l+1)$ by Corollary 7.6. Also its characteristic polynomial $\chi(c \mathscr{A}, t)$ is equal to $(t-1) \Pi_{i=1}^{l}\left(t-b_{i}\right)$. Therefore $\chi(\mathscr{A}, t)=\Pi_{i=1}^{l}\left(t-b_{i}\right)$ by [6, Proposition 2.51, Definition 2.52]. Apply Theorem 7.10. 
CoRollary 7.13. If $\mathscr{A}$ is a 2-arrangement, then $\left.H^{p}\left(\Omega_{\nabla}^{*}\right)\right)=0(p \neq 2)$ and

$$
\operatorname{dim} H^{2}\left(\Omega_{\nabla}^{*}\right)=|\chi(\mathscr{A}, 1-r)|
$$

for every $\mathscr{A}$-transverse polynomial $f$ of degree $r>0$.

Proof. If $l=2$, then $c \mathscr{A}$ is a central 3 -arrangement. Apply Corollary 7.8 and Theorem 7.10.

Example 7.14. Let an affine arrangement $\mathscr{A}$ be defined by

$$
Q=\prod_{i=1}^{l} x_{i} \prod_{i=1}^{l}\left(x_{i}+1\right) \prod_{i=1}^{l}\left(x_{i}-1\right) \prod_{1 \leq i \leq j \leq l}\left(x_{i}+x_{j}\right) \prod_{1 \leq i \leq j \leq l}\left(x_{i}-x_{j}\right) .
$$

Then $c \mathscr{A}$ is a Coxeter arrangement of type $B_{l+1}$ whose exponents are $\{1,3,5, \ldots$, $2 l+1\}$. Therefore $\mathscr{c A}$ is free (e.g., [6, Theorem 6.60]). By Corollary 7.12, $H^{p}\left(\Omega_{\nabla}^{*}\right)=0(p \neq l)$ and

$$
\operatorname{dim} H^{l}\left(\Omega_{\nabla}^{*}\right)=\prod_{i=1}^{l}(2 i+1+q)
$$

for every $\mathscr{A}$-transverse polynomial $f$ of degree $r>0$.

\section{REFERENCES}

[1] Aomoto, K., Les équations aux différences linéaires et les intégrales des fonctions multiformes, J. Fac. Sci. Univ. Tokyo, Sec. IA, 22 (1975), 271-297.

[2] - On the structure of integrals of power products of linear functions, Sci. Papers, Coll. Gen. Educ. Univ. Tokyo, 27 (1977), 49-61.

[ 3 ] - Hypergeometric functions, the past, today, and... (from complex analytic view point). (in Japanese) in Sûgaku, 45 (1993), 208-220.

[ 4 ] Aomoto, K., Kita, M., Orlik, P., Terao, H., Twisted de Rham cohomology groups of logarithmic forms, to appear in Advances in Math.

[ 5 ] Gelfand, I. M., Zelevinsky, A. V., Algebraic and combinatorial aspects of the general theory of hypergeometric functions, Funct. Anal. and Appl., 20 (1986), $183-197$.

[6] Orlik, P., Terao, H., Arrangements of hyperplanes. Grundlehren der math. Wiss. 300, Springer-Verlag, Berlin-Heidelberg-New York, 1992.

[ 7 ] - Arrangements and Milnor fibers, Math. Ann., 301 (1995), 211-235.

[8] Saito, K., Theory of logarithmic differential forms and logarithmic vector fields, J. Fac. Sci. Univ. Tokyo Sec. 1A, 27 (1980), 266-291.

[9] Solomon, L., Terao, H., A formula for the characteristic polynomial of an arrangement, Adv. in Math., 64 (1987), 305-325.

[10] Terao, H., Generalized exponents of a free arrangement of hyperplanes and Shephard-Todd-Brieskorn formula, Invent. math., 63 (1981), 159-179. 
[11] Yuzvinsky, S., First two obstructions to the freeness of arrangements, Trans. AMS, 335 (1993), 231-244.

[12] Varchenko, A., Multidimensional hypergeometric functions and the representation theory of Lie algebras and quantum groups, World Scientific Publishers, 1995.

[13] Ziegler, G., Combinatorial construction of logarithmic differential forms. Adv. in Math., 76 (1989), 116-154.

H. Terao

Mathematics Department

University of Wisconsin

Madison, WI 53706

S. Yuzvinsky

Department of Mathematics

University of Oregon

Eugene, OR 97403 